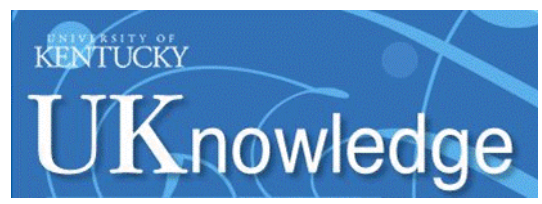

University of Kentucky

UKnowledge

\title{
Caffeine Consumption in a Long-Term Psychiatric Hospital: Tobacco Smoking May Explain in Large Part the Apparent Association Between Schizophrenia and Caffeine Use
}

\author{
Manuel Arrojo-Romero \\ Gallegan Health System, Spain \\ Carmen Armas Barbazán \\ Gallegan Health System, Spain \\ Javier D. López-Moriñigo \\ King's College London, UK \\ Ramón Ramos-Rios \\ Gallegan Health System, Spain \\ Manuel Gurpegui \\ University of Granada, Spain \\ Follow this and additional works at: https://uknowledge.uky.edu/psychiatry_facpub \\ see next of the forsychiatry and Perychology Commons \\ Right click to open a feedback form in a new tab to let us know how this document benefits you.
}

\section{Repository Citation}

Arrojo-Romero, Manuel; Barbazán, Carmen Armas; López-Moriñigo, Javier D.; Ramos-Rios, Ramón; Gurpegui, Manuel; Martinez-Ortega, José M.; Jurado, Dolores; Diaz, Francisco J.; and de Leon, Jose, "Caffeine Consumption in a Long-Term Psychiatric Hospital: Tobacco Smoking May Explain in Large Part the Apparent Association Between Schizophrenia and Caffeine Use" (2015). Psychiatry Faculty Publications. 30.

https://uknowledge.uky.edu/psychiatry_facpub/30

This Article is brought to you for free and open access by the Psychiatry at UKnowledge. It has been accepted for inclusion in Psychiatry Faculty Publications by an authorized administrator of UKnowledge. For more information, please contact UKnowledge@lsv.uky.edu. 


\section{Caffeine Consumption in a Long-Term Psychiatric Hospital: Tobacco Smoking May Explain in Large Part the Apparent Association Between Schizophrenia and Caffeine Use}

Digital Object Identifier (DOI)

http://dx.doi.org/10.1016/j.schres.2015.01.042

Notes/Citation Information

Published in Schizophrenia Research, v. 164, issue 1-3, p. 234-241.

(C) 2015 Elsevier B.V.

This manuscript version is made available under the CC-BY-NC-ND 4.0 license http://creativecommons.org/licenses/by-nc-nd/4.0/

\section{Authors}

Manuel Arrojo-Romero, Carmen Armas Barbazán, Javier D. López-Moriñigo, Ramón Ramos-Rios, Manuel Gurpegui, José M. Martinez-Ortega, Dolores Jurado, Francisco J. Diaz, and Jose de Leon 
Caffeine Consumption in a Long-Term Psychiatric Hospital: Tobacco Smoking May Explain in Large Part the Apparent Association between Schizophrenia and Caffeine Use

Manuel Arrojo-Romero, Carmen Armas Barbazán, Javier D. LópezMoriñigo, Ramón Ramos-Ríos, Manuel Gurpegui, José M. MartínezOrtega, Dolores Jurado, Francisco J. Diaz, Jose de Leon

(C) 2015 Elsevier B.V. This manuscript version is made available under the CC-BY-NC-ND 4.0 license http://creativecommons.org/licenses/bync-nd/4.0/ 
Words in text: 3,516

Caffeine consumption in a long-term psychiatric hospital: tobacco smoking may explain in large part the apparent association between schizophrenia and caffeine use

Manuel Arrojo-Romero ${ }^{a}$, Carmen Armas Barbazán ${ }^{b}$, Javier D. López-Moriñigo ${ }^{c}$, Ramón RamosRíos $^{\mathrm{a}}$, Manuel Gurpegui ${ }^{\mathrm{de}}$, José M. Martínez-Ortega ${ }^{\mathrm{d}, \mathrm{e}}$, Dolores Jurado,f, Francisco J. Diaz ${ }^{\mathrm{g}}$, Jose de Leon ${ }^{\text {eh }}$

aPsychiatry Department (EOXI de Santiago), Gallegan Health System (Servicio Gallego de

Salud), Santiago de Compostela, Spain

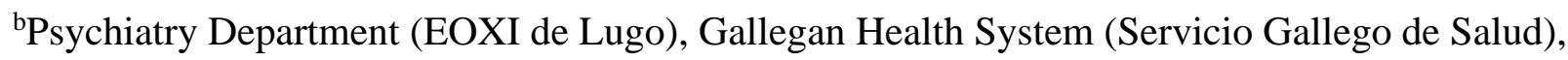
Lugo, Spain

${ }^{\mathrm{c}}$ Department of Psychosis Studies, Institute of Psychiatry, King's College London, London, UK.

Servicio de Psiquiatría. EOXI Santiago. Servicio Gallego de Salud.

${ }^{\mathrm{d} D e p a r t m e n t ~ o f ~ P s y c h i a t r y, ~ U n i v e r s i t y ~ o f ~ G r a n a d a, ~ G r a n a d a, ~ S p a i n . ~}$

${ }^{\mathrm{e}}$ Institute of Neurosciences, Center for Biomedical Research, University of Granada, Granada, Spain.

fDepartment of Preventive Medicine and Public Health, University of Granada, Granada, Spain.

gDepartment of Biostatistics, The University of Kansas Medical Center, Kansas City, Kansas, U.S.A.

${ }^{\mathrm{h}}$ Mental Health Research Center at Eastern State Hospital, Lexington, Kentucky, U.S.A.

Corresponding author and reprints: Jose de Leon, M.D., UK Mental Health Research Center at Eastern State Hospital, 1350 Bull Lea Road, Lexington, KY 40511. Phone (859) 246-8440. Fax (859) 246-8446. E-mail: jdeleon@uky.edu 


\begin{abstract}
This study further explores the association between schizophrenia and caffeine use by combining two prior published Spanish samples (250 schizophrenia outpatients and 290 controls from the general population) with two Spanish long-term inpatient samples from the same hospital (145 with schizophrenia and 64 with other severe mental illnesses). The specific aims were to establish whether or not, after controlling for confounders including tobacco smoking, the association between schizophrenia and caffeine is consistent across schizophrenia samples and across different definitions of caffeine use. The frequency of caffeine use in schizophrenia inpatients was not significantly higher than that in non-schizophrenia inpatients $(77 \%, 111 / 145$ vs. $75 \%, 48 / 64$ ) or controls but was significantly higher than in schizophrenia outpatients. The frequency of high caffeine users among caffeine users in schizophrenia inpatients was not significantly higher than in non-schizophrenia inpatients $(45 \%, 50 / 111$ vs. $52 \%, 25 / 48)$ or controls, but was significantly lower than in schizophrenia outpatients. Smoking was significantly associated with caffeine use across all samples and definitions. Between 2-3\% of schizophrenia inpatients, schizophrenia outpatients and non-schizophrenia inpatients showed caffeinism (>700 mg/day in smokers). Several of these smoking patients with caffeinism were also taking other inducers, particularly omeprazole. The lack of consistent association between schizophrenia and caffeine use is surprising when compared with the very consistent association between tobacco smoking and caffeine use across all of our analyses (use and high use in users) and all our samples. The confounding effects of tobacco smoking may explain in large part the apparent association between schizophrenia and caffeine use.
\end{abstract}

Keywords: caffeine; case-control study; drug interactions, omeprazole; schizophrenia; smoking. 


\section{Introduction}

Caffeine probably causes most of its biological effects by blocking all adenosine receptors located at the neurons and brain glial cells and removing the brain's adenosinergic tonus (Ribeiro and Sebastião, 2010). Animal models suggest that 1) adenosine plays a role opposite to dopamine in the striatum; 2) adenosine agonists produce effects similar to dopamine antagonists; and 3) adenosine antagonists, such as caffeine, produce effects similar to increased dopaminergic neurotransmission (Ferré, 1997).

Schneier and Siris (1987) suggested that schizophrenia was associated with increased rates of caffeine consumption. Later, Hughes et al. (1998) described that patients with schizophrenia appeared to have high caffeine intake but studies were small and not completely consistent. These old studies did not control for the confounding factor that schizophrenia is strongly and consistently associated with tobacco smoking (de Leon and Diaz, 2005) and tobacco smoking is also associated with caffeine (Istvan and Mattarazzo, 1984; Gurpegui et al., 2007). Furthermore, tobacco smoke compounds induce caffeine metabolism by increasing the expression of the cytochrome P450 1A2 (CYP1A2). Heavy smokers need two to three times higher caffeine intake to get the same serum caffeine concentrations as non-smokers (de Leon et al., 2003). Other CYP1A2 inducers are some antiepileptic drugs (carbamazepine or phenytoin) and omeprazole (de Leon et al. 2005).

In two consecutive studies, Gurpegui et al. (2004; 2006) explored the relationship between schizophrenia and caffeine intake after controlling for tobacco smoking. First, current caffeine intake was associated with current smoking and alcohol use in 250 schizophrenia outpatients (Gurpegui et al., 2004). Among caffeine consumers, the amount of consumed caffeine was associated with smoking but not with schizophrenia symptomatology measured 
cross-sectionally. In an extension study, the schizophrenia outpatient sample was compared with 290 controls (Gurpegui et al., 2006). Current caffeine intake was significantly less frequent in schizophrenia after controlling for potential confounding factors. Among caffeine consumers, high caffeine consumption was significantly more frequent in schizophrenia outpatients than in the control group.

In our previous studies we did not pay attention to "caffeinism", the extremely high consumption of caffeine in schizophrenia. The literature only provides anecdotal reports of “caffeinism" but Williams and Ghandi (2008) have proposed that caffeine intake >700 mg/day may be a sign of this "caffeinism."

If animal pharmacological models are correct in proposing a close relationship between the adenosine system and dopamine, then one should find a strong and consistent relationship between schizophrenia and caffeine in the clinical environment. The lack of definitive proof of an association between caffeine intake and schizophrenia is surprising, since schizophrenia is definitively associated with excessive use of nicotine and water.

There is a strong and consistent association between schizophrenia and tobacco smoking (de Leon and Diaz, 2012). When compared with the general population, schizophrenia patients definitively have increased daily smoking initiation and decreased smoking cessation. When compared with people with other severe mental illnesses (SMIs), this association is less evident; schizophrenia is only associated with a smaller increase of daily smoking initiation but no differences in smoking cessation. Vulnerability to schizophrenia may be associated with an increased risk for starting daily smoking (de Leon, 1996; de Leon and Diaz, 2012). Wing et al. (2012) reviewed the proposed neurobiological mechanisms behind the association between smoking and schizophrenia. 
Polydipsia, an excessive intake of water and other fluids, is associated with schizophrenia after controlling for confounders such as smoking, chronicity or medications. This association has been demonstrated in institutionalized patients using SMIs as controls (de Leon et al., 1994; 1996; 2002), but it is less evident in less severe and less chronic patients (de Leon et al., 1994). Although schizophrenia appears to be consistently associated with excessive use of nicotine and water, it is not clear that it is consistently associated with excessive use of caffeine. Moreover, a hepatic pharmacokinetic mechanism may explain why heavy smokers with schizophrenia consume more caffeine so that the same quantity of caffeine reaches their brains. In the extension study (Gurpegui et al., 2006), we demonstrated two significant associations between caffeine and schizophrenia, one negative (fewer caffeine users) and one positive (more high-intake users within caffeine users), but we did not compare them with people with other SMIs, who are better controls for schizophrenia patients.

This study further explores the association between schizophrenia and caffeine use by combining the two prior published Spanish samples (250 schizophrenia outpatients and 290 controls) with two additional Spanish samples of long-term inpatients from the same hospital: 145 with schizophrenia and 64 with other SMIs sharing similar environmental influences including psychiatric medications. The specific aims were to establish, after controlling for confounders including tobacco smoking, whether or not the association between schizophrenia and caffeine is consistent across schizophrenia samples and across different operational definitions of caffeine use (using versus not using caffeine, high intake in caffeine users and total daily caffeine consumption in caffeine users).

\section{Subjects and methods}

\subsection{Subjects}


All inpatients from a long-term psychiatric hospital (Hospital Psiquiátrico de Conxo, Santiago de Compostela, Spain) were cross-sectionally investigated for the prevalence of caffeine intake and its quantity. DSM-IV diagnoses were made by the treating psychiatrist after reviewing all medical records available, which include many years of observation (Table 1). There were 111 schizophrenia inpatients and 48 with other SMIs. Similarly to the outpatient sample, this new sample included schizoaffective disorder. The study was approved by an ethics committee and patients signed a written informed consent.

The previously-published Spanish schizophrenia sample (see footnote g of Table 2) included 250 stable outpatients (Gurpegui et al., 2004; 2006). The previously-published 258 Spanish controls were recruited at a family medicine outpatient clinic (see footnote i of Table 2) and appeared to be a reasonable representation of the general population (Gurpegui et al., 2006).

\subsection{Access to caffeine and tobacco}

There is almost unlimited access to caffeinated beverages and tobacco in this urban hospital. The main limiting factor is the money available to each patient from their disability pensions and/or family. The hospital cafeteria tends to be cheaper than surrounding businesses and provides caffeinated coffee in two sizes and caffeinated colas in three different sizes. Patients can walk to surrounding areas and buy caffeinated beverages from shops and/or consume coffee in nearby cafeterias. The nursing staff, who has worked with these patients for many years, knows these businesses very well, is aware of the interactions between the patients and surrounding community, and attempts to keep them as smooth as possible. No patient consumed tea beverages, but this is a cultural choice.

Nursing staff kept patients' cigarettes. These cigarettes were bought in large quantities using the patients' and/or family's money. Patients could also buy more expensive cigarettes in the 
hospital cafeteria or outside the hospital. In summary, by law, patients are free to smoke as much as they desire, but the prohibition against smoking during some hospital activities and the need to ask the nursing staff for cigarettes probably contributed to lengthening the time between cigarettes.

\subsection{Procedures}

As in the prior studies (Gurpegui et al., 2004; 2006), the consumption of caffeinated beverages, tobacco, alcohol and illegal drugs was surveyed. Caffeine intake was assessed by treating physicians when they considered the patient most likely to cooperate. A standardized questionnaire was used to record the number of coffee beverages and caffeinated colas consumed per week. It differed from the outpatient questionnaire in three minor ways. First, the inpatient questionnaire did not ask about tea since nobody consumed it; second, the questions about the size of the beverages were easier to answer since the hospital used standard sizes; and third, nurses who had known the patients for years were available to help the psychiatrists make better clinical judgments rather than blindly trusting patients' information. Caffeine intake in mg/day was computed by employing previously used correction factors (see footnote e, Table 1). In caffeine users, a high amount of caffeine intake was defined as a caffeine intake $\geq 200 \mathrm{mg} /$ day (Gurpegui et al., 2005). Smoking assessments used the same definitions as before (Gurpegui et al., 2005; see Table 1, footnote b) and included the Fagerström Test for Nicotine Dependence (FTND) (Heatherton et al., 1991) which required the patient to answer some subjective questions. To establish the number of cigarettes used daily, the psychiatrist also consulted the nursing staff members who controlled most of the cigarettes. The history of alcohol and illegal drug abuse or dependence was assessed by treating psychiatrists using all available information. Patients could not use alcohol or illegal drugs on hospital grounds. 
For the first time, we used the definition of caffeinism as a caffeine intake $>700 \mathrm{mg} /$ day for smokers (Williams and Ghandi, 2008). As non-smokers have approximately half the caffeine metabolic capacity that smokers have (de Leon et al., 2003), a definition of caffeinism in nonsmokers as a caffeine intake $>350 \mathrm{mg} /$ day was explored.

\subsection{Statistical analysis}

Total caffeine intake in $\mathrm{mg} /$ day was used as the dependent variable in gamma regression models with a log link that analyzed only caffeine users (Table 2). Log-gamma regression models were conducted with Stata 13 (StataCorp LP, College Station, TX). They fit well according to residual analyses and the link test.

The association between schizophrenia and the dichotomous dependent variable, caffeine use or no caffeine use, was explored in univariate analyses using ORs with their 95\% CI from two-way cross-tabulations (footnote a of Table 3). Then a logistic regression model was built by starting with the independent variables that provided a significant $\mathrm{p}$-value in univariate analyses (Table 3, upper panel). A similar set of univariate analyses and a logistic regression analysis, plus the addition of inducer use as an independent variable, were performed in caffeine consumers by using a high amount of caffeine intake ( $\geq 200 \mathrm{mg} /$ day) as the dependent variable (Table 4, upper panel). Analogous analyses compared inpatients with schizophrenia versus outpatients with schizophrenia, and inpatients with schizophrenia versus controls from the general population (Tables 3 and 4, middle and lower panels). Logistic regressions were performed with the Statistical Package for the Social Sciences (SPSS 21). All models fit well according to the Hosmer-Lemeshow goodness-of-fit test (Hosmer and Lemeshow, 2000).

\section{Results}

\subsection{Sample description and daily caffeine intake}


Table 1 describes patients and their daily caffeine intake

\subsection{Log-gamma regression in individuals consuming caffeine}

In the inpatient sample, schizophrenia had no significant effects on daily caffeine intake (Table 2, footnote e). After adjusting for the number of cigarettes smoked and comparing with the general population, schizophrenia was not significantly associated with daily caffeine intake, either in schizophrenia inpatients (Table 2, third panel, footnotes $\mathrm{j}$ and $\mathrm{k}$ ) or in schizophrenia outpatients (Table 2, fourth panel, footnotes 1 and $\mathrm{m}$ ). After controlling for confounders, the number of cigarettes smoked was associated with increased caffeine intake in four analyses (Table 2, four panels). Taking inducers had a significant effect size in inpatients $(42.2 \%$, Table 2 , upper panel).

\subsection{Caffeinism}

In the smokers of the inpatient sample, there were 4 schizophrenia and 1 nonschizophrenia patients consuming $>700 \mathrm{mg}$ /day of caffeine (Table 5). Three of these 5 patients were also taking omeprazole, which might contribute to a greater induction and the need for higher caffeine doses. In the outpatient schizophrenia sample, there were 7 smokers consuming $>700 \mathrm{mg} /$ day of caffeine. The caffeinism prevalences were around $3 \%$ in all 3 SMI samples and $0 \%$ in the Spanish controls (see Table 5, lower panel and footnote b).

Our attempt to explore a definition of caffeinism as consuming $>350 \mathrm{mg} / \mathrm{day}$ in nonsmoking inpatients indicated that 3 of 4 subjects were probably consuming high quantities of caffeine to compensate for omeprazole (Table 5).

\subsection{Comparing prevalences of caffeine use in schizophrenia inpatients vs. inpatients with SMI}

There was no significant difference between schizophrenic inpatients vs. SMI inpatients in the prevalence of caffeine use $\left(77 \%, 111 / 145\right.$ vs. $75 \%, 48 / 64$, respectively; Pearson $\chi^{2}=0.06$; 
$\mathrm{p}=0.81 ; \mathrm{OR}=1.1$, CI 0.55-2.2). Significant variables were male sex, current smoking and history of alcohol abuse/dependence in the univariate analyses, and male sex and current smoking in the logistic regression (Table 3, upper panel).

3.5. Comparing prevalences of caffeine use in schizophrenia inpatients vs. schizophrenia outpatients

There was a significant difference between schizophrenia inpatients and schizophrenia outpatients in the prevalence of caffeine use $(77 \%, 111 / 145$, versus 59\%, 147/250, respectively; unadjusted $\mathrm{p}<0.001)$. After adjusting for potential confounders, this difference remained significant $(\mathrm{OR}=3.5, \mathrm{p}<0.001)$ and male sex, current smoking and inpatient status were also significant (Table 3, middle panel).

3.6. Comparing prevalences of caffeine use in schizophrenia inpatients vs. controls

Schizophrenia inpatients $(77 \%, 111 / 145)$ did not differ significantly from controls who came from the general population $(70 \%, 204 / 290)$ in their likelihood of being caffeine consumers (unadjusted $\mathrm{OR}=1.4, \mathrm{p}=0.17)$, not even after adjusting for potential confounders $(\mathrm{p}=0.57)$. Significant variables in the logistic regression were male sex and current smoking (Table 3, lower panel).

3.7. Comparing prevalences of high caffeine use among caffeine users in schizophrenia inpatients vs. inpatients with SMI

Among patients currently consuming caffeine, a comparison of schizophrenia inpatients versus SMI inpatients showed no significant difference in the prevalences of high caffeine intake $\left(45 \%, 50 / 111\right.$ vs. $52 \%, 25 / 48$, respectively; Pearson $\chi^{2}=0.7 ; \mathrm{p}=0.41 ; \mathrm{OR}=0.75$, CI 0.38-1.5). The significant variables in the univariate analyses and the logistic regression were current smoking and taking inducers (Table 4, upper panel). 
3.8. Comparing prevalences of high caffeine use among caffeine users in schizophrenia inpatients vs. schizophrenia outpatients

Schizophrenia inpatients who consumed caffeine were significantly less likely to be highlevel caffeine consumers than schizophrenia outpatients who consumed caffeine $(45 \%, 50 / 111$, versus $64 \%$, 94/147, respectively; unadjusted $\mathrm{p}=0.002$ ). This difference remained significant after controlling for potential confounders (adjusted $\mathrm{OR}=0.47, \mathrm{p}=0.004$ ). Male sex and current smoking were also significant (Table 4, middle panel).

3.9. Comparing prevalences of high caffeine use among caffeine users in schizophrenia inpatients vs. the general population

Inpatients with schizophrenia who were caffeine consumers $(45 \%, 50 / 111)$ did not significantly differ from caffeine consumers from the general population $(36 \%, 73 / 204)$ in their likelihood of being high-intake caffeine consumers (unadjusted $\mathrm{OR}=1.47, \mathrm{p}=0.11$ ), not even after adjusting for current smoking $(\mathrm{p}=0.9)$. Only current smoking was significant (Table 4 , lower panel).

\section{Discussion}

4.1. Lack of consistency in the association between schizophrenia and prevalence of caffeine use

The first study aim was to establish the stability of the association of schizophrenia with prevalence of caffeine use after controlling for confounders. We found that schizophrenia inpatients had no significantly greater frequency of caffeine use than non-schizophrenia inpatients or controls from the general population. The schizophrenia inpatient sample had significantly greater frequency of caffeine use than the schizophrenia outpatients, who also had significantly lower frequency than the controls (Gurpegui et al., 2006). 
4.2. Lack of consistency in the association between schizophrenia and prevalence of high caffeine use among caffeine users

The second study aim was to establish the stability of the association of schizophrenia with high caffeine use among caffeine users after controlling for confounders. We found that schizophrenia inpatients had no significantly greater frequency of high caffeine use among caffeine users than did non-schizophrenia inpatients or controls from the general population. Moreover, schizophrenia inpatients had a significantly lower frequency than schizophrenia outpatients after controlling for confounding variables. Our clinical experience suggests that average schizophrenia outpatients may have more access to caffeine and tobacco products. Our data supported greater daily access to cigarettes and caffeine in outpatient smokers compared to inpatient smokers, although access to caffeine by caffeine users was not greater in outpatient non-smokers than in inpatient non-smokers (Table 6).

\subsection{Caffeine intake and caffeinism}

Caffeine intake was not associated with schizophrenia when compared with controls or other SMI inpatients (Table 2). The limited published information suggests that the extreme use of very high levels of caffeine, or caffeinism, may be particularly associated with schizophrenia (Williams and Gandhi, 2008; Meredith et al., 2013). Our two surveys indicate that a small number of schizophrenia patients (3\%) may consistently show caffeinism, defined as $>700$ $\mathrm{mg} / \mathrm{day}$. Our inpatient survey also identified a patient with caffeinism and psychotic disorder NOS and a history of polysubstance abuse. Several of these patients with caffeinism were taking omeprazole, indicating that additive inductive effects of omeprazole with smoking may explain some caffeinism cases. Better surveys in the future should measure serum caffeine levels and control for medications with inductive effects. 


\subsection{Consistency of the association between tobacco smoking and caffeine use}

The lack of a consistent association between schizophrenia and caffeine use is surprising when compared with the very consistent association between tobacco smoking and caffeine use across all of our analyses. After controlling for confounders, current smoking was significantly associated with increased prevalence of caffeine use in all three analyses comparing schizophrenia inpatients with SMI inpatients, schizophrenia outpatients and controls. Similarly, in the three analyses and after controlling for confounders, current smoking was significantly associated with increased prevalence of high caffeine use in caffeine users and with higher caffeine intake in caffeine users. Therefore, smoking is very strongly and consistently associated with caffeine use and high caffeine use, and this association may be an important component of the apparent association between schizophrenia and caffeine use.

\subsection{Limitations}

Although our surveys have greater strengths than prior studies due to the use of relatively large samples of systematically recruited patients and careful control of smoking and other confounding factors, they are not free of limitations. The samples were studied cross-sectionally and not followed longitudinally. Conducting longitudinal studies in schizophrenia and obtaining funding for them is not easy. The control sample of inpatients with other SMIs was very heterogeneous but reflected the usual pattern of admissions in this Spanish long-term hospital. Our experience with US state hospitals is similar (de Leon et al.,1996; 2002). Most of the longterm inpatients have schizophrenia and those who do not have schizophrenia are a heterogeneous patient mix.

In the inpatient sample, we had no ratings on schizophrenia symptoms or extrapyramidal symptoms. However, we have serious doubts that caffeine has a strong association with either 
schizophrenia or extrapyramidal symptoms. In the outpatient sample, the correlations between caffeine intake and PANSS scores, long-term prognosis or daily antipsychotic intake were very low $<0.10$ in both the total sample and in caffeine users. Undoubtedly the schizophrenia inpatients included patients with illnesses of much greater chronicity and severity than the schizophrenia outpatients. We think that that greater severity and/or chronicity in schizophrenia would not influence caffeine intake in any way, but we have to acknowledge that we have no way of correcting for that difference.

This study included only one outpatient schizophrenia sample that had a significantly lower prevalence of caffeine use than both the schizophrenia inpatient and general population control samples. Therefore, we do not know if that lower prevalence is specific to the schizophrenia outpatient sample or whether it can be generalized to other schizophrenia outpatient samples. We are convinced that this is not due to lack of access to caffeine (see Table 6).

Omeprazole (and less frequently other antiepileptic inducers) appeared to contribute to high caffeine intake in the inpatients but this could not be examined in the outpatient sample. The most obvious limitation was the lack of collection of serum caffeine samples to definitively establish when there is high caffeine use and to better control for the effects of smoking and other inducers in caffeine metabolism. Caffeine has a relatively short half-life. To better establish the relationship between caffeine intake and metabolism one can average morning and afternoon serum caffeine concentrations (de Leon et al. 2003), or also measure serum concentrations of paraxanthine, a longer-lasting metabolite (Perera et al. 2011). The lack of serum caffeine levels has to be placed in the context of the very few published studies reporting them in schizophrenia patients. In a study of patients trying to quit smoking, Ghandi et al. (2010) found higher serum 
caffeine levels in 104 smokers with schizophrenia (2722 ng/mL) compared with 63 smokers without any mental illness (1122 ng/mL).

Our schizophrenia outpatients had access to alcohol and demonstrated that alcohol and caffeine use were significantly associated. On the other hand, in caffeine users, alcohol was associated with less frequent high caffeine consumption among non-smokers, probably because alcohol may potentiate caffeine brain pharmacodynamic effects (Gurpegui et al., 2006). Chronic alcohol intake decreases adenosine tone (Ferré and O'Brien, 2011), suggesting that alcohol users may have decreased need for higher caffeine intake since lower caffeine intake may have the same effects in the brain for a chronic alcohol user as high caffeine intake has for a non-alcohol user. As our inpatients had no access to alcohol, we could not replicate the possibility of this pharmacodynamic interaction between alcohol and caffeine in non-smokers (Gurpegui et al., 2006).

In conclusion, the apparent association between schizophrenia and caffeine use is not particularly strong or consistent after controlling for confounders or using different controls. In particular, this association may be partly explained by confounding with smoking. Schizophrenia does not appear to be consistently associated with an increased prevalence of caffeine use and the increased prevalence of high caffeine intake in caffeine users does not stand up when controlling for confounders. Omeprazole and antiepileptic inducers, and particularly smoking, contribute to the excessive caffeine intake in schizophrenia. Future epidemiological studies, preferably using serum concentrations, will need to establish whether caffeinism is particularly prevalent in schizophrenia or not, and determine the contribution of inducers. 


\section{References}

Baldessarini, R.J., 1985. Chemotherapy in Psychiatry. Harvard University Press, Cambridge, MA.

de Leon, J., 1996. Smoking and vulnerability for schizophrenia. Schizophr. Bull. 22, 405-409.

de Leon, J., Armstrong, S.C., Cozza, K.L, 2005. The dosing of atypical antipsychotics.

Psychosomatics 46, 262-273.

de Leon, J., Dadvand, M., Canuso, C., Odom-White, A., Stanilla, J., Simpson, G.M., 1996. Polydipsia and water intoxication in a long-term psychiatric hospital. Biol. Psychiatry 40, 28-34.

de Leon, J., Diaz, F.J., Rogers, T., Browne, D., Dinsmore, L., Ghosheh, O., Dwoskin, L.P., Crooks, P., 2003. A pilot study of plasma caffeine concentrations in a US sample of smoker and non-smoker volunteers. Prog. Neuropsychopharmacol. Biol. Psychiatry 27, 165-171.

de Leon, J., Diaz, F.J., 2005. A meta-analysis of worldwide studies demonstrates an association between schizophrenia and tobacco smoking behaviors. Schizophr. Res. 76, 135-157.

de Leon, J., Diaz, F.J., 2012. Genetics of schizophrenia and smoking: an approach to studying their comorbidity based on epidemiological findings. Hum. Genet. 131, 877-901.

de Leon, J., Tracy, J., McCann, E., McGrory, A., 2002. Polydipsia and schizophrenia in a psychiatric hospital: A replication study. Schizophr. Res. 57, 293-301.

de Leon, J., Verghese, C., Tracy, J., Josiassen, R.C., Simpson, G.M., 1994. Polydipsia and water intoxication in psychiatric patients: A review of the epidemiological literature. Biol. Psychiatry. 35, 408-419. 
Ferré, S., 1997. Adenosine-dopamine interactions in the ventral striatum: implications for the treatment of schizophrenia. Psychopharmacology. 133, 107-120.

Ferré S, O'Brien, M.C., 2011. Alcohol and caffeine: the perfect storm. J. Caffeine Res. 1,153162.

Gandhi, K.K., Williams, J.M., Menza, M., Galazyn, M., Benowitz, N.L., 2010. Higher serum caffeine in smokers with schizophrenia compared to smoking controls. Drug Alcohol Depend. 110, 151-155.

Gurpegui, M., Aguilar, M.C., Martínez-Ortega, J.M., Diaz, F.J., de Leon, J., 2004. Caffeine intake in outpatients with schizophrenia. Schizophr. Bull. 30, 935-945.

Gurpegui, M., Aguilar, M.C., Martínez-Ortega, J.M., Jurado, D., Diaz, F.J., Quintana, H.M., de Leon, J., 2006. Fewer but heavier caffeine consumers in schizophrenia: a case-control study. Schizophr. Res. 86, 276-283.

Gurpegui, M., Jurado, D., Luna, J.D., Fernandez-Molina, C., Moreno-Abril, O., Galvez, R., 2007. Personality traits associated with caffeine intake and smoking. Prog. Neuropsychopharmacol. Biol. Psychiatry. 31, 997-1005.

Heatherton, T.F., Kozlowski, L.T., Frecker, R.C., Fagerström, K.O., 1991. The Fagerström Test for Nicotine Dependence: a revision of the Fagerström Tolerance Questionnaire. Br. J. Addict. 86, 1119-1127.

Hosmer, D.W., Lemeshow, S., 2000. Applied Logistic Regression. 2nd ed, John Wiley \& Sons, Inc,, New York.

Hughes, J.R., McHugh, P., Holtzman, S., 1998. Caffeine and schizophrenia. Psychiatr. Serv. 49, 1415-1417. 
Istvan, J., Matarazzo, J.D., 1984. Tobacco, alcohol and caffeine use: a review of their interrelationships. Psychol. Bull. 95, 301-326.

Kahn, R.S., Fleischhacker, W.W., Boter, H., Davidson, M., Vergouwe, Y., Keet, I.P., Gheorghe, M.D., Rybakowski, J.K., Galderisi, S., Libiger, J., Hummer, M., Dollfus, S., López-Ibor, J.J., Hranov, L.G., Gaebel, W., Peuskens, J., Lindefors, N., Riecher-Rössler, A., Grobbee, D.E.; EUFEST study group, 2008. Effectiveness of antipsychotic drugs in first-episode schizophrenia and schizophreniform disorder: an open randomised clinical trial. Lancet. $371,1085-1097$.

Mencías, E., Mayero, L.M., 2000. Manual de Toxicología Básica. Díaz de Santos, Madrid, Spain.

Meredith, S.E., Juliano, L.M., Hughes, J.R., Griffiths, R.R., 2013. Caffeine use disorder: a comprehensive review and research agenda. J. Caffeine Res. 3, 114-130.

Perera, V., Gross, A.S., Xu, H., McLachlan, A.J., 2011. Pharmacokinetics of caffeine in plasma and saliva, and the influence of caffeine abstinence on CYP1A2 metrics. J, Pharm, Pharmacol. 63, 1161-1168.

Ribeiro, J.A., Sebastião, A.M., 2010. Caffeine and adenosine. J. Alzheimers. Dis. 20 (Suppl 1), S3-S15.

Rey, M.J., Schulz, P., Costa, C., Dick, P., Tissot, R., 1989. Guidelines for the dosage of neuroleptics. I: Chlorpromazine equivalents of orally administered neuroleptics. Int. Clin. Psychopharmacol. 4, 95-104.

Schulz, P., Rey, M.J., Dick, P., Tissot, R., 1989. Guidelines for the dosage of neuroleptics. II: Changing from daily oral to long acting injectable neuroleptics. Int. Clin. Psychopharmacol. 4, 105-114. 
Schneier, F.R., Siris, S.G., 1987. A review of psychoactive substance use and abuse in schizophrenia. Patterns of drug choice. J. Nerv. Ment. Dis. 175, 641-652.

Williams, JM, Gandhi, K.K., 2008. Use of caffeine and nicotine in people with schizophrenia. Curr Drug Abuse Rev. 1,155-161.

Wing, V.C., Wass, C.E., Soh, D.W., George, T.P. 2012. A review of neurobiological vulnerability factors and treatment implications for comorbid tobacco dependence in schizophrenia. Ann. NY. Acad. Sci.1248, 89-106.

Woods, S.W., 2003. Chlorpromazine equivalent doses for the newer atypical antipsychotics. J. Clin. Psychiatry. 64, 663-667. 
Table 1. Description of the sample

\begin{tabular}{|c|c|c|}
\hline \multirow[t]{2}{*}{ Variables } & Schizophrenia inpatients & Inpatients with other SMIs ${ }^{\mathrm{a}}$ \\
\hline & PERCENTAGES & \\
\hline$\widehat{o}$ gender & $68 \%(98 / 145)$ & $69 \%(44 / 64)$ \\
\hline Age (>37 years) & $90 \%(130 / 145)$ & $86 \%(55 / 64)$ \\
\hline College education & $5 \%(7 / 145)$ & $3 \%(2 / 64)$ \\
\hline Current smoking ${ }^{\mathrm{b}}$ & $63 \%(92 / 145)$ & $69 \%(44 / 64)$ \\
\hline History of alcohol abuse/dependence & $19 \%(28 / 145)$ & $42 \%(27 / 64)$ \\
\hline Taking any inducer ${ }^{\mathrm{c}}$ & $37 \%(54 / 145)$ & $56 \%(36 / 64)$ \\
\hline Omeprazole & $34 \%(49 / 145)$ & $50 \%(32 / 64)$ \\
\hline \multirow[t]{2}{*}{ Carbamazepine or phenytoin } & $4 \%(6 / 145)$ & $9 \%(6 / 64)$ \\
\hline & $\mathrm{MEAN} \pm \mathrm{SD}$ & \\
\hline Age in years & $56.1 \pm 14.4$ & $52.1 \pm 14.0$ \\
\hline Hospitalization in years & $13.5 \pm 15.1$ & $7.1 \pm 11.1$ \\
\hline FTND (in smokers) & $4.3 \pm 2.6(\mathrm{~N}=92)$ & $4.2 \pm 2.6(\mathrm{~N}=43)^{\mathrm{d}}$ \\
\hline Daily number of cigarettes (in smokers) & $25.5 \pm 11.4(\mathrm{~N}=92)$ & $23.6 \pm 11.0(\mathrm{~N}=44)$ \\
\hline Chlorpromazine equivalents $^{\mathrm{e}}$ & $1897 \pm 1348$ & $1246 \pm 1393$ \\
\hline Caffeine intake $(\mathrm{mg} / \text { day })^{\mathrm{f}}$ & MEDIAN $\left(25^{\text {th }} \mathrm{P}, 75^{\text {th }} \mathrm{I}\right.$ & \\
\hline All patients ${ }^{\mathrm{h}}$ & $130(53,265) \quad \mathrm{N}=145$ & $150(9,300) \mathrm{N}=64$ \\
\hline Caffeine users & $182(107,300) \mathrm{N}=111^{\mathrm{i}}$ & $219(119,362) \mathrm{N}=48^{\mathrm{j}}$ \\
\hline$\hat{\sigma}$ caffeine users & $183(110,311) \mathrm{N}=86$ & $254(126,380) \mathrm{N}=38$ \\
\hline o caffeine users & $150(72,239) \quad \mathrm{N}=25$ & $160(107,300) \mathrm{N}=10$ \\
\hline Smoking caffeine users & $203(113,312) \mathrm{N}=85$ & $238(135,369) \mathrm{N}=40$ \\
\hline$\widehat{o}$ smoking caffeine users & $203(126,329) \mathrm{N}=74$ & $283(150,406) \mathrm{N}=31$ \\
\hline q smoking caffeine users & $196(107,265) \mathrm{N}=11$ & $160(107,230) \mathrm{N}=9$ \\
\hline Non-smoking caffeine users & $115(53,203) \quad \mathrm{N}=26$ & $150(107,289) \mathrm{N}=8$ \\
\hline$\widehat{o}$ non-smoking caffeine users & $115(54,157) \quad \mathrm{N}=12$ & $150(107,256) \mathrm{N}=7$ \\
\hline onon-smoking caffeine users & $128(53,238) \quad \mathrm{N}=14$ & $\mathrm{~N}=1$ \\
\hline Caffeine users: smoking and inducers & $261(149,362) \mathrm{N}=32$ & $300(160,380) \mathrm{N}=22$ \\
\hline$\widehat{c}$ caffeine users: smoking and inducers & $256(145,363) \mathrm{N}=31$ & $314(176,439) \mathrm{N}=16$ \\
\hline q caffeine users: smoking and inducers & $\mathrm{N}=1$ & $160(147,314) \mathrm{N}=6$ \\
\hline Caffeine users: smoking or inducers & $183(107,280) \mathrm{N}=65$ & $183(109,343) \mathrm{N}=24$ \\
\hline o caffeine users: smoking or inducers & $176(107,304) \mathrm{N}=48$ & $205(119,368) \mathrm{N}=20$ \\
\hline caffeine users: smoking or inducers & $196(99,239) \quad \mathrm{N}=17$ & $128(107,262) \mathrm{N}=4$ \\
\hline Caffeine users: no inducers & $111(53,150) \quad \mathrm{N}=14$ & $128(107)$ \\
\hline$\widehat{o}$ caffeine users: no inducers & $115(53,150) \quad \mathrm{N}=7$ & $128(107)$ \\
\hline 9 caffeine users: no inducers & $107(53,150) \quad \mathrm{N}=7$ & \\
\hline
\end{tabular}

FTND: Fagerström Test for Nicotine Dependence. P: percentiles. SD: standard deviation. SMI: severe mental illness.

${ }^{a}$ All inpatients who had no schizophrenia (or schizoaffective disorder) were included in this group of other SMIs. Primary diagnoses were: $27 \%$ of patients (17/64) had severe personality disorders; $17 \%(11 / 64)$ delusional disorders; $15 \%$ (10/64) substance use disorders; $14 \%(9 / 64) \operatorname{mood}$ disorders; $14 \%$ (9/64) mental retardation; 10\% (7/64) psychotic disorders not otherwise specified; and $2 \%(1 / 64)$ obsessive-compulsive disorder.

${ }^{\mathrm{b}}$ All current smokers were daily cigarette smokers; the lowest daily number of cigarettes was 5 . None of the patients used other tobacco products. 
${ }^{\mathrm{c}}$ Taking inducers means taking omeprazole, carbamazepine or phenytoin.

${ }^{\mathrm{d}}$ One patient did not cooperate in FTND scoring.

${ }^{\mathrm{e}}$ Chlorpromazine equivalents were calculated by combining oral and long-acting antipsychotics (Baldessarini, 1985; Rey et al., 1985; Schulz et al, 1989; Mencias and Mayero, 2000; Woods, 2003; and Kahn et al., 2008).

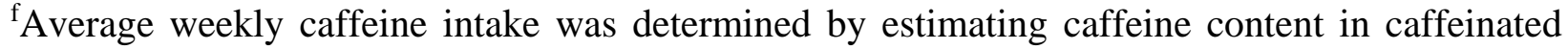
beverages in those patients who reported taking caffeine on a regular basis every week; then the weekly estimation was converted into $\mathrm{mg} / \mathrm{day}$. The standard caffeine content utilized was 100 $\mathrm{mg}$ in a $150 \mathrm{cc}$ cup of drip coffee; $45 \mathrm{mg}$ in a cup of tea; and, for caffeinated sodas, $23 \mathrm{mg}$ in a $200 \mathrm{cc}$ glass of soda (Gurpegui et al., 2004). In this hospital tea was not available.

'Daily caffeine intake followed a non-normal distribution; thus, we described it using medians (and $25^{\text {th }}$ and $75^{\text {th }}$ percentiles).

historted by non-caffeine users. As smoking, taking inducers and gender may influence caffeine metabolism, medians after stratification are described to allow comparison with future published samples.

${ }^{i}$ Among 111 schizophrenia caffeine users, 91\% (101/111) consumed coffee and 51\% (57/111). consumed caffeinated colas.

${ }^{j}$ Among 48 non-schizophrenia caffeine users, 90\% (43/48) consumed coffee and 44\% (21/48) consumed caffeinated colas. 
Table 2. Analysis of total daily caffeine intake (mg/day) in those consuming caffeine: significant variables in univariate ${ }^{\mathrm{a}}$ analyses, and in multivariate analyses using log-gamma regression. ${ }^{\text {b }}$

\begin{tabular}{|c|c|c|c|c|c|c|c|}
\hline & $\mathrm{B}^{\mathrm{c}}$ & $95 \% \mathrm{CI}$ & $\mathrm{p}$ & Adjusted $\mathrm{B}^{\mathrm{c}}$ & $95 \% \mathrm{CI}$ & $\mathrm{p}$ & Effect size $^{\mathrm{d}} \quad 95 \% \mathrm{CI}$ \\
\hline \multicolumn{8}{|c|}{ INPATIENTS WITH SCHIZOPHENIA (N=111) VS. THOSE WITH SMI (N=48) } \\
\hline Number of cigarettes & 0.0208 & $(0.0121,0.0294)$ & $<0.001$ & 0.0218 & $(0.0135,0.0230)$ & $<0.001$ & $+2.20 \%(1.36,3.05)$ \\
\hline Male sex & 0.414 & $(0.078,0.751)$ & 0.02 & -- & -- & -- & -- \\
\hline Taking inducers ${ }^{\mathrm{f}}$ & 0.316 & $(0.034,0.598)$ & 0.03 & 0.352 & $(0.106,0.599)$ & 0.005 & $+42.2 \%(11.1,82.0)$ \\
\hline \multicolumn{8}{|c|}{ INPATIENTS WITH SCHIZOPHRENIA (N=111) VS. OUTPATIENTS WITH SCHIZOPHRENIAg $(\mathrm{N}=147)^{\mathrm{h}}$} \\
\hline Number of cigarettes & 0.0203 & $(0.0136,0.0270)$ & $<0.001$ & 0.0221 & $0.0157,0.0285)$ & $<0.001$ & $+2.24 \%(1.58,2.89)$ \\
\hline Age $>37 \mathrm{y}$ & -- & -- & -- & 0.348 & $(0.125,0.571)$ & 0.002 & $+41.6 \%(13.3,77.0)$ \\
\hline \multicolumn{8}{|c|}{ INPATIENTS WITH SCHIZOPHRENIA (N=111) VS. CONTROLS FROM GENERAL POPULATION $(\mathrm{N}=204)^{\mathrm{j}}$} \\
\hline Number of cigarettes & 0.0263 & $(0.0201,0.0325)$ & $<0.001$ & 0.0263 & $0201,0.0325)$ & $<0.001$ & $+2.66 \%(2.03,3.30)$ \\
\hline Male sex & 0.284 & $(0.0760,0.492)$ & 0.007 & -- & & -- & -- \\
\hline College education & -0.282 & $(-0.496,-0.069)$ & 0.01 & -- & & -- & -- \\
\hline Schizophrenia & $0.528^{\mathrm{k}}$ & $(0.325,0.730)$ & $<0.001$ & -- & & -- & -- \\
\hline Age $>37 \mathrm{y}$ & 0.328 & $(0.116,0.539)$ & 0.002 & -- & & -- & -- \\
\hline \multicolumn{8}{|c|}{ OUTPATIENTS WITH SCHIZOPHRENIA (N=147) VS. GENERAL POPULATION $(\mathrm{N}=204)^{1}$} \\
\hline Number of cigarettes & 0.0238 & $(0.0183,0.0294)$ & $<0.001$ & 0.0236 & $0.0184,0.0289)$ & $<0.001$ & $+2.39 \%(1.86,2.93)$ \\
\hline Male sex & 0.290 & $(0.0616,0.519)$ & 0.01 & -- & - & -- & -- \\
\hline College education & -0.257 & $(-0.495,-0.0192)$ & 0.03 & -- & - & -- & -- \\
\hline Schizophrenia & $0.640^{\mathrm{m}}$ & $(0.441,0.839)$ & $<0.001$ & -- & -- & -- & -- \\
\hline Age $>37 y$ & 0.277 & $(0.0528,0.501)$ & 0.02 & 0.253 & $0.0733,0.433)$ & 0.006 & $+28.8 \%(7.6,54.2)$ \\
\hline
\end{tabular}

${ }^{a}$ The independent variables used to build log-gamma regression models were number of cigarettes; male sex $(1=$ male, $0=$ female); college education ( $1=$ having college education, 0 otherwise); schizophrenia ( $1=$ schizophrenia, 0 otherwise); age $>37$ years $(1$ if age $>37$ years, 0 otherwise); history of alcohol abuse or dependence ( 1 if subject has history of alcohol abuse or dependence, 0 otherwise); and taking inducers ( 1 if taking carbamazepine, phenytoin or omeprazole, 0 otherwise). Two variables, history of alcohol abuse and taking inducers, were not available in the outpatient schizophrenia and normal control samples from Gurpegui et al. (2006).

${ }^{\mathrm{b}}$ Total caffeine intake in $\mathrm{mg} /$ day had a distribution that was right-skewed with a relatively heavy tail. Thus a log-gamma regression was appropriate. The log-gamma regression model with a log link that analyzed only caffeine users allowed including the fact that there were subjects with very high caffeine consumption relative to the rest of the subjects. In practice, in this study, a log-gamma regression model can be interpreted as the usual linear regression model with a log-transformed total caffeine intake as the dependent variable. However, the distribution of the errors is not the normal distribution but the gamma distribution, which is always right-skewed.

${ }^{c} \mathrm{~B}=$ regression coefficient. 
${ }^{\mathrm{d}}$ An adjusted effect size was computed as $(\exp (\mathrm{B})-1) \times 100$. For the variable, number of cigarettes smoked, the effect size is interpreted as the percent increase in caffeine intake per one-cigarette increase in the number of cigarettes smoked. For dichotomous independent variables, the effect size is interpreted as the percent increase in caffeine intake when comparing subjects with a value of 1 versus subjects with a value of 0 in the independent variable. For instance, the effect size of taking inducers in the first panel, $+42.2 \%$, suggests that caffeine intake in subjects who were on inducers was $42.2 \%$ higher than that in subjects who were not on inducers.

${ }^{\text {e} A d j u s t e d ~ r e g r e s s i o n ~ c o e f f i c i e n t s ~(B) ~ a n d ~ e f f e c t ~ s i z e s ~ w e r e ~ c o m p u t e d ~ w i t h ~ t h e ~ f i n a l ~ l o g-g a m m a ~ r e g r e s s i o n ~ m o d e l ~ w h i c h ~ i n c l u d e d ~}$ number of cigarettes and taking inducers as independent variables. After controlling for potential confounders, there were no significant differences in total caffeine intake between schizophrenia inpatients who were caffeine consumers and inpatients with other severe mental illnesses who were caffeine consumers $(\mathrm{p}=0.6)$.

${ }^{\mathrm{f}}$ Taking omeprazole, carbamazepine or phenytoin.

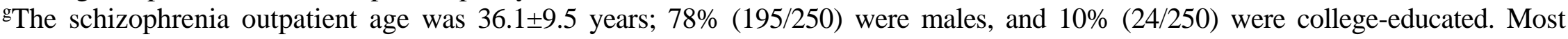
schizophrenia outpatients $(81 \%, 203 / 250)$ had previously been hospitalized at least once. The mean \pm standard deviation of number of hospitalizations was $4.1 \pm 4.9$. Most of the hospitalizations were short-term. In summary, these patients were clearly less chronic and less severely ill than the schizophrenia inpatients.

${ }^{\mathrm{h}}$ Adjusted regression coefficients (B) and effect sizes were computed with the final log-gamma regression model which included number of cigarettes and age $>37$ years as independent variables. After controlling for potential confounders, there were no significant differences in total caffeine intake between schizophrenia inpatients who were caffeine consumers and schizophrenia outpatients who were caffeine consumers $(\mathrm{p}=0.3)$.

'The controls' mean age was 40.5 \pm 15.1 years; 39\% (113/290) were males and 49\% (141/290) had a college education.

jOnly the number of cigarettes smoked was found to have a significant effect on total daily caffeine intake in the multivariate loggamma regression model that included the other investigated independent variables and after a backward variable selection.

${ }^{\mathrm{k}}$ The unadjusted effect size of schizophrenia on daily caffeine intake was $+69.6 \%(38.4,107.5)$. After adjusting for current smoking, the effect size continued to be significant and was $+39.6 \%(14.5,70.2), p=0.001$. But after adjusting for the number of cigarettes instead of adjusting for current smoking, the effect size was lower and not significant, $+20.1 \%(-1.6,46.5)$, $\mathrm{p}=0.07$. Thus, not only current smoking but heavy smoking accounts for the association between schizophrenia and caffeine consumption.

${ }^{1}$ Only the number of cigarettes smoked and age $>37$ years were found to have a significant effect on total daily caffeine intake in the multivariate log-gamma regression model that included the other investigated independent variables and after a backward variable selection.

mThe unadjusted effect size of schizophrenia on daily caffeine intake was $+89.7 \%(55.4,131.4)$. After adjusting for both current smoking and age $>37$ years, the effect size continued to be significant and was $+42.3 \%(15.6,75.1)$, $\mathrm{p}=0.001$. But after adjusting for both the number of cigarettes (instead of current smoking) and age $>37$ years, the effect size was lower and not significant, $+16.2 \%(-$ 
$6.8,44.9), \mathrm{p}=0.2$. Thus, not only current smoking but heavy smoking accounts for the association between schizophrenia and caffeine consumption. 
Table 3. Caffeine intake: significant variables in the univariate ${ }^{\mathrm{a}}$ and multivariate analyses

\begin{tabular}{|c|c|c|c|c|c|c|c|c|}
\hline & OR & $95 \% \mathrm{CI}$ & $\chi^{2}$ & $\mathrm{p}$ & Adjusted OR & $95 \% \mathrm{CI}$ & Wald $\chi^{2}$ & p \\
\hline \multicolumn{9}{|c|}{ INPATIENTS WITH SCHIZOPHENIA (N=145) VS. THOSE WITH OTHER SEVERE MENTAL ILLNESSES (N=64) } \\
\hline Male sex & 6.3 & $3.1-12.5$ & 30.8 & $<0.001$ & $3.6^{1}$ & 1.7-7.9 & 10.6 & 0.001 \\
\hline Current smoking & 13.03 & $6.0-28.1$ & 53.6 & $<0.001$ & $8.8^{1}$ & $3.9-20.3$ & 26.4 & $<0.001$ \\
\hline Alcohol abuse/dependence (history) & 4.1 & $1.5-11.0$ & 9.0 & 0.003 & -- & -- & -- & -- \\
\hline \multicolumn{9}{|c|}{ INPATIENTS WITH SCHIZOPHRENIA $(\mathrm{N}=145)$ VS. OUTPATIENTS WITH SCHIZOPHRENIA $(\mathrm{N}=250)^{\mathrm{c}}$} \\
\hline Male sex & 3.01 & 1.94 .8 & 22.5 & $<0.001$ & 2.4 & $1.4 \quad 4.2$ & 10.8 & $<0.001$ \\
\hline t smoking & 5.0 & 3.2 & 51.5 & $<0.001$ & 4.8 & $2.9-7.8$ & 37.8 & $<0.001$ \\
\hline Inpatient status & 2.3 & $1.4-3.6$ & 12.8 & $<0.001$ & 3.5 & $2.0-5.9$ & 20.6 & $<0.001$ \\
\hline \multicolumn{9}{|c|}{ INPATIENTS WITH SCHIZOPHRENIA (N=145) VS. CONTROLS FROM THE GENERAL POPULATION $(\mathrm{N}=290)^{\mathrm{d}}$} \\
\hline Male sex & 2.5 & $1.6-3.9$ & 17.0 & $<0.001$ & 2.0 & $1.3-3.2$ & 8.7 & 0.003 \\
\hline Current smoking & 3.4 & 2.15 .4 & 26.8 & $<0.001$ & 2.9 & $1.8-4.7$ & 18.2 & .001 \\
\hline College education & 1.8 & 1.12 .9 & 6.0 & 0.014 & -- & -- & -- & -- \\
\hline ge $>37 y$ & 0.49 & $0.31-0.78$ & 9.2 & 0.002 & -- & -- & -- & -- \\
\hline
\end{tabular}

andependent variables for univariate analysis using cross tabulations with caffeine use or not as the dependent variable were schizophrenia illness, current daily smoking, history of alcohol abuse or dependence, sex, age >37 years, and college level of education (Gurpegui et al., 2006).

${ }^{\mathrm{b}}$ Adjusted ORs were computed with the final logistic regression model which included male sex and current smoking as independent variables (Hosmer-Lemeshow goodness-of-fit test $\chi^{2}=3.0 ; \mathrm{df}=4 ; \mathrm{p}=0.56$ ).

${ }^{\mathrm{c}}$ Adjusted ORs were computed with the final logistic regression model which included male sex, current smoking and inpatient status as independent variables (Hosmer-Lemeshow goodness-of-fit test $\chi^{2}=5.22 ; \mathrm{df}=4 ; \mathrm{p}=0.27$ ).

${ }^{\mathrm{d}}$ Adjusted ORs were computed with the final logistic regression model which included male sex and current smoking as independent variables (Hosmer-Lemeshow goodness-of-fit test $\chi^{2}=2.46 ; \mathrm{df}=2 ; \mathrm{p}=0.29$ ). 
Table 4. High caffeine intake in those consuming caffeine: significant variables in the univariate ${ }^{\mathrm{a}}$ and multivariate analyses

\begin{tabular}{|c|c|c|c|c|c|c|c|c|}
\hline & OR & $95 \% \mathrm{CI}$ & $\chi^{2}$ & $\mathrm{p}$ & Adjusted OR & $95 \% \mathrm{CI}$ & Wald $\chi^{2}$ & $\mathrm{p}$ \\
\hline \multicolumn{9}{|c|}{ INPATIENTS WITH SCHIZOPHENIA (N=111) VS. THOSE WITH OTHER SEVERE MENTAL ILLNESSES $(\mathrm{N}=48)^{\mathrm{b}}$} \\
\hline nt smoking & 2.6 & 1.1-5.9 & 5.5 & 0.019 & 3.0 & $1.53-7.0$ & 6.5 & 0.011 \\
\hline Taking inducers ${ }^{\mathrm{c}}$ & 2.3 & $1.2-4.3$ & 6.6 & 0.01 & 2.6 & 1.3-5.0 & 7.7 & 0.005 \\
\hline \multicolumn{9}{|c|}{ INPATIENTS WITH SCHIZOPHRENIA $(\mathrm{N}=111)$ VS. OUTPATIENTS WITH SCHIZOPHRENIA $(\mathrm{N}=147)^{\mathrm{d}}$} \\
\hline oking & 3.4 & $1.8-6.6$ & 15.2 & $<0.001$ & 3.4 & $1.8-6.5$ & 13.5 & $<0.001$ \\
\hline npatient status & 0.46 & $0.28-0.76$ & 9.2 & 0.002 & 0.47 & $0.28-0.79$ & 8.2 & 0.004 \\
\hline College education & 2.7 & $0.97-7.7$ & 3.8 & 0.05 & -- & -- & -- & -- \\
\hline
\end{tabular}

$\begin{array}{cccccc}\text { College education } & 2.7 & 0.97-7.7 & 3.8 & 0.05 & --\end{array}$

Current smoking

$\begin{array}{llll}3.08 & 1.9-5.0 & 22.1 & <0.001\end{array}$

$--$

$--$

${ }^{a}$ Independent variables for univariate analysis using cross tabulations with high caffeine use or not within caffeine users as the dependent variable were schizophrenia illness, current daily smoking, history of alcohol abuse or dependence, gender, age $>37$ years, and college level of education (Gurpegui et al., 2006). Taking inducers was added as an independent variable in the first sample including schizophrenia and non-schizophrenia inpatients.

${ }^{\mathrm{b}}$ Adjusted ORs were computed with the final logistic regression model which included current smoking and taking inducers as independent variables (Hosmer-Lemeshow goodness-of-fit test $\chi^{2}=3.5 ; \mathrm{df}=2 ; \mathrm{p}=0.18$ ).

${ }^{\mathrm{c}}$ Taking omeprazole, carbamazepine or phenytoin.

${ }^{\mathrm{d}}$ Adjusted ORs were computed with the final logistic regression model which included current smoking and inpatient status as independent variables (Hosmer-Lemeshow goodness-of-fit test $\chi^{2}=0.29 ; \mathrm{df}=2 ; \mathrm{p}=0.87$ ). 
Table 5. Caffeinism ( $>700 \mathrm{mg} /$ day in patients induced by smoking or $>350 \mathrm{mg} /$ day in non-smokers)

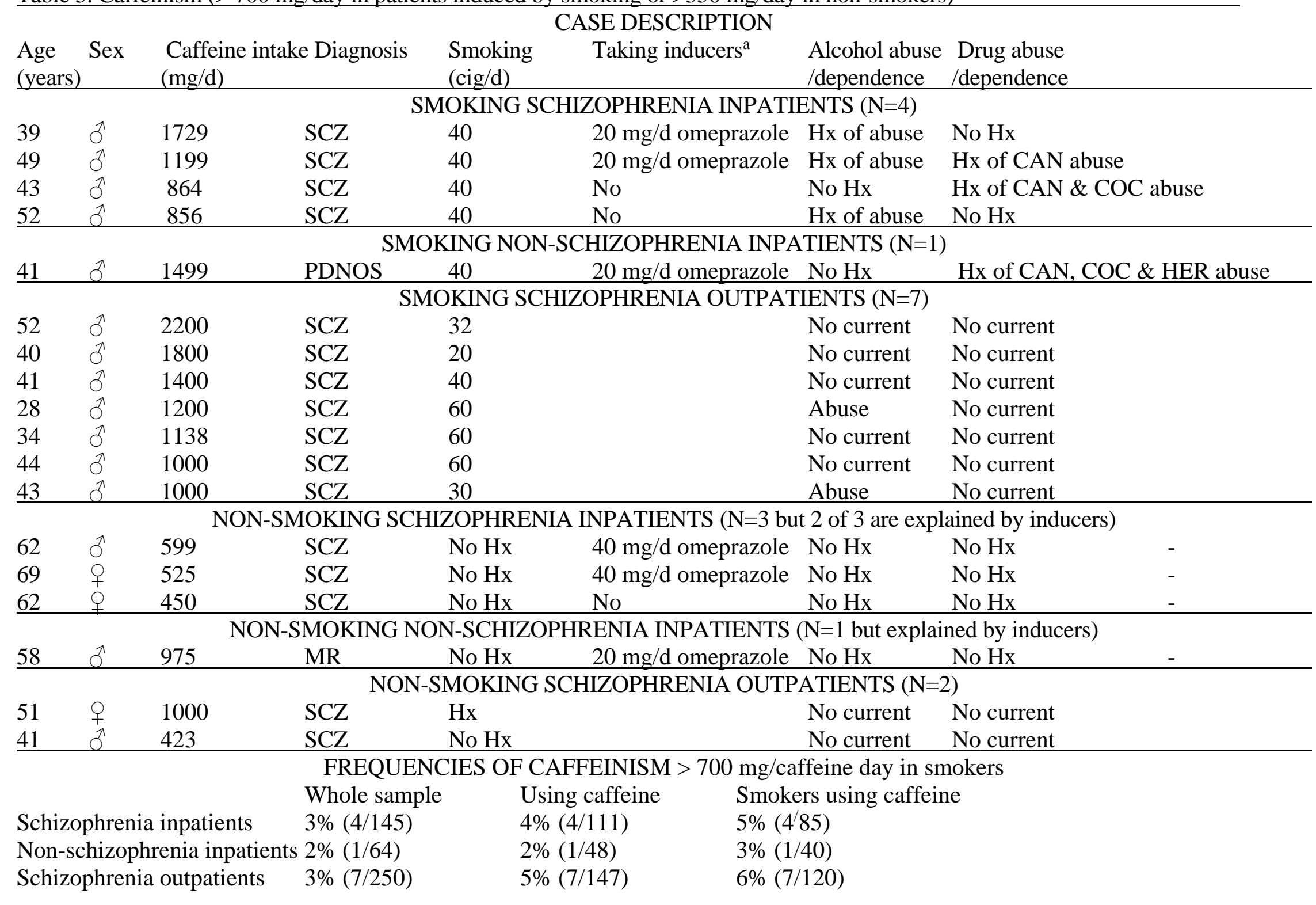


Spanish controls $0 \%(0 / 290)^{\mathrm{b}}$

$0 \%(0 / 204)$ $0 \%(0 / 78)$

CAN: cannabis; COC: cocaine; HER: heroin; Hx: history; MR: mental retardation; PDNOS: psychotic disorder NOS; SCZ: schizophrenia.

${ }^{\text {a }}$ The schizophrenia outpatient sample had no data on omeprazole intake.

${ }^{\mathrm{b}}$ The definition of caffeinism as consuming $>700 \mathrm{mg} /$ day in smokers and $>350 \mathrm{mg} /$ day in non-smokers appears reasonable in our sample since no Spanish control met the criterion (highest daily intakes were $643 \mathrm{mg}$ in smokers and $326 \mathrm{mg}$ in non-smokers). 
Table 6. Median intakes to explore access to tobacco and caffeine products in schizophrenia patients

\begin{tabular}{lll}
\hline & $\begin{array}{l}\text { Inpatients } \\
\text { Median }(\mathrm{N})\end{array}$ & $\begin{array}{l}\text { Outpatients } \\
\text { Median }(\mathrm{N})\end{array}$ \\
\hline Number of daily cigarettes in smokers & $20(92)$ & $30(173)$ \\
Caffeine in mg/day in caffeine users & & \\
$\quad$ Total sample & $182.5(111)$ & $200(147)$ \\
Smokers & $203.1(85)$ & $238(120)$ \\
$\quad$ Non-smokers & $115 \quad(26)$ & $100(27)$ \\
\hline
\end{tabular}

